\title{
Adaptive Functioning, Social Skills, Mental Health, and Personal Strengths among Adolescents with Prenatal Alcohol Exposure (PAE)
}

\author{
Jennifer Mariasine ${ }^{1}$, Jacqueline Pei ${ }^{1}$, Cheryl Poth ${ }^{1}$, Dorothy Henneveld ${ }^{2} \&$ Carmen Rasmussen ${ }^{3}$ \\ ${ }^{1}$ Department of Educational Psychology, Faculty of Education University of Alberta, Canada \\ ${ }^{2}$ Catholic Social Services, Edmonton, Alberta, Canada \\ ${ }^{3}$ Department of Pediatrics, Faculty of Medicine and Dentistry, University of Alberta, Canada \\ Correspondence: Jennifer Mariasine, Graduate Student, Department of Educational Psychology, University of \\ Alberta, Edmonton, Alberta, T6G 2G5, Canada. E-mail: jennifer.mariasine@ualberta.ca
}

Received: July 26, 2013

doi:10.5539/ijps.v6n2p36
Accepted: March 24, 2014 Online Published: May 20, 2014

URL: http://dx.doi.org/10.5539/ijps.v6n2p36

\begin{abstract}
Three key areas of dysfunction reported as having a significant impact on how adolescents with Prenatal Alcohol Exposure (PAE) navigate their teen years and work towards independence are: mental health, social skills, and adaptive functioning. However, little is known about how adolescents with PAE perceive their own functioning relative to others, and what they and others see as potential strengths on which they may build. Participants included 32 adolescents (17 male, 15 female); $31 \%$ had confirmed prenatal exposure to alcohol (PAE) and the remaining $69 \%$ had a diagnosis of a Fetal Alcohol Spectrum Disorder (FASD). Adolescents and their caregivers completed the following measures: ABAS-2 (adaptive skills), SSIS (social skills), BASC-2 (mental health functioning), and BERS-2 (personal strengths). Overall, caregivers rated adolescents as having significantly more difficulties across all measures assessed when compared to the adolescents. The only area of agreement between caregiver and adolescents prenatally exposed to alcohol was on career strength. The knowledge gained from the results of this exploratory study can help adolescents with PAE better understand themselves and help researchers to better characterize this population.
\end{abstract}

Keywords: PAE, adolescents, caregiver, perceptions, strengths, adaptive skills, mental health, social skills

\section{Introduction}

Fetal Alcohol Spectrum Disorder (FASD) is an umbrella term referring to the array of physical, cognitive, behavioral, and/or learning deficits found in children, adolescents, and adults whose mothers drank alcohol during pregnancy (Chudley et al., 2005). Consistently, researchers looking at clinical and community samples, as well as caregivers, have found that individuals prenatally exposed to alcohol may have significant neurobehavioral impairments. In particular, deficits are reported in executive functioning (Rasmussen, 2005), learning, memory, language, intelligence, motor, visual-spatial abilities (see Kodituwakku, 2007, 2009; Mattson, Crocker, \& Nguyen, 2011), and academic achievement (Streissguth et al., 1994). Problems with disrupted school experiences, mental health problems, incarceration and retention in the justice system, as well as inappropriate sexual behaviors and alcohol and drug abuse have also been described (Streissguth, 1997). The resulting financial costs of supporting individuals impacted by FASD are enormous. In Canada, the annual cost of supporting persons 0 to 53 years of age with FASD is estimated to be $\$ 5.3$ billion (Stade et al., 2009).

FASD is a descriptive rather than a diagnostic term and includes the following diagnoses: Fetal Alcohol Effects (FAE), Fetal Alcohol Syndrome (FAS), Alcohol-Related Birth Defects (ARBD), and Alcohol-Related Neurodevelopmental Disorder (ARND) (Streissguth, \& O'Malley, 2000). Due to the complexities surrounding the diagnosis of an FASD, those who have been exposed prenatally to alcohol but where there is not enough information to warrant a diagnosis of an FASD, are described as having Prenatal Alcohol Exposure (PAE). Both adolescents who have a confirmed diagnosis of an FASD and those who have confirmed PAE but no diagnosis of an FASD, are described in this paper.

Three key areas of dysfunction are reported as having a significant impact on how adolescents navigate their teen years and work towards independence: mental health, social skills, and adaptive functioning (Rasmussen, Andrew, Zwaigenbaum, \& Tough, 2008). Mental health problems are a serious issue among individuals with 
FASD, with mental health comorbidity rates as high as $90 \%$ with the most common being depression, mood and anxiety disorders, Attention Deficit Hyperactivity Disorder (ADHD), and Conduct Disorder (CD) (see Pei, Denys, Hughes, \& Rasmussen, 2011 for a review). Moreover, these mental health issues appear to be lifelong spanning from childhood (O'Connor, 2001; O'Connor et al., 2002) to adolescence and adulthood (Barr et al., 2006; Spohr \& Steinhausen, 1987; Steinhausen \& Spohr, 1998).

Social skills deficits are also common among children, adolescents, and adults with PAE with significant impairments documented in social relationships (see Kully-Martens, Denys, Treit, Tamana, \& Rasmussen, 2012 for a review). These social skill deficits may become more pronounced with age (Thomas, Kelly, Mattson, \& Riley, 1998; Whaley, O'Connor, \& Gunderson, 2001). In particular, individuals with FASD may also misinterpret social cues (Green, 2007), which can alter their perception of a social situation, which can alter the self-concept of an adolescent with PAE and the way in which others perceive them.

Adaptive behavior consists of skills an individual uses to function in daily life in order to meet the daily demands of their environment, including such tasks as personal care and interacting with other people. Adaptive functioning deficits are common among individuals with FASD (Ase et al., 2012; Kaemingk \& Paquette, 1999; Streissguth et al., 2004). They have difficulty with employment and independent living (Spohr, Willms, \& Steinhausen, 2007), which can result in many affected individuals dependent on external support (Steinhausen \& Spohr, 1998). One study found the adaptive skills of adolescents and adults with FASD to be at the level of a 7-year-old, and most prominent in the area of socialization (Streissguth et al., 1991). When compared to children with ADHD, the socialization and communication skills of children with FASD failed to improve with age (Crocker, Vaurio, Riley, \& Mattson, 2009).

The deficits in mental health, social skills and adaptive functioning have a significant impact on how adolescents with PAE deal with their transition to adulthood, and continue to impact them well into adulthood. Yet little is known about how adolescents perceive their own functioning relative to others, and what they and others see as potential strengths on which they may build. Foster parents of children with FASD reported that one of their motives for fostering was to help children focus on their strengths (Olson, Oti, Gelo, \& Beck, 2009). However, this is an aspiration goal and not one that has been empirically evaluated in research.

Therefore we sought to investigate the perceptions of adolescents with PAE and their caregivers as they pertain to three critical areas of deficit (adaptive functioning, mental health, and social skills functioning) and areas of strength. Adolescents with FASD are often a difficult group to engage and retain in research and intervention, and clinically described concerns with high attrition and frequent drop-outs from programs illuminates the possibility that we do not fully understand the perception of this group in a way that fosters motivation and engagement. Consideration of both the perceived challenges and strengths within this population is essential if we are to shape interventions that better support adolescents in increasing their self-motivation to seek out help that will aid in successfully transitioning to adulthood. Increasing understanding of the different perspectives of youth and their caregivers can help us anticipate possible miscommunication and disengagement in support services and intervention efforts, and demonstrate increased responsiveness to the individuals involved.

\section{Method}

\subsection{Sample}

Participants included 32 adolescents (17 male and 15 female) with a mean age of 16 years and 9 months (range 13 years 6 months to 20 years 3 months) who either had confirmed PAE with no FASD diagnosis or an FASD diagnosis. The youth were recruited through a community youth mentoring program. Not all 32 adolescents completed all questionnaires, consequently analyses were conducted with fewer subjects (range between 19 and 29 adolescents depending on the questionnaire). Ten of the participants had confirmed PAE with no diagnosis and 22 had an FASD related diagnosis. Of the 22 participants with FASD, 9 were diagnosed with FASD, 6 with Alcohol-Related Neurodevelopment Disorder (ARND), 4 with Fetal Alcohol Syndrome (FAS), 2 with FASD, Static Encephalopathy, and 1 with Fetal Alcohol Effects (FAE). Diagnoses of an FASD were made using Astley and Clarren's 4-digit diagnostic grid, on which growth, facial features, brain functioning, and gestational alcohol exposure are independently ranked on a four-point Likert scale (see Astley, 2004). All FASD diagnoses were confirmed through medical records to ensure that each participant had a formal diagnosis of an FASD using accepted diagnostic methods (e.g. Chudley et al., 2005). Exclusion criteria included participants who did not have confirmed PAE or a validated FASD diagnosis.

There were several comorbid diagnoses present for adolescents in the study. Among the adolescents with confirmed PAE, comorbid diagnoses included (n): Attention Deficit Hyperactivity Disorder (ADHD; $N=3$ ), Oppositional Defiant Disorder (ODD; $\mathrm{N}=2$ ), and Reactive Attachment Disorder (RAD; $N=2$ ). Among the 
adolescents with FASD, comorbid diagnoses included (n): ADHD $(\mathrm{N}=15)$, ODD $(\mathrm{N}=8)$, depression $(\mathrm{N}=5)$, RAD $(\mathrm{N}=4)$, seizures $(\mathrm{N}=4)$, bipolar disorder $(\mathrm{N}=2)$, and anxiety disorder $(\mathrm{N}=2)$.

\subsection{Measures}

Measures of adaptive behavior (ABAS-2), social skills (SSIS), mental health (BASC-2), and personal strengths (BERS-2) were routinely administered as part of a community youth program. The adolescent, and/or their caregiver completed the scales when they entered the program. Consent was obtained from participant adolescents and their caregiver to use this data for research purposes.

\subsubsection{The Adaptive Behavior Assessment System, Second Edition (ABAS-2)}

The ABAS-2 (Harrison \& Oakland, 2003) is designed to specifically measure adaptive skills across the lifespan that have primary relevance for an individual's functioning in the home and community. It consists of 5 different rater forms: Parent/Primary Caregiver (for children aged 0-5), Teacher/Daycare Provider (for children aged 2-5), Parent (for children aged 5-21), Teacher (for children aged 5-21) and Adult (aged 16-89). For the purpose of this study we used the Parent (for children aged 5-21) and Adult (aged 16-89) forms. The Parent form is written at a $5^{\text {th }}$ grade reading level and the Adult form at approximately a $6^{\text {th }}-7^{\text {th }}$ grade reading level. Adaptive behavior is made up of the skills an individual uses to function in daily life, including such tasks as personal care and interacting with other people. The ABAS-2 measured daily living skills (what adolescents actually do, or can do, without the assistance of others) by asking a series of questions that were answered on a 4 point Likert scale $(0=$ is not able to perform the task, $1=$ never or almost never performs the task, $2=$ performs the task sometimes, or $3=$ always or almost always performs the task. The raters also indicated whether their response was a guess or an estimate for each item. ABAS-2 yields scaled scores, which are derived from the raw scores of each of the skill areas. These scaled scores are used to derive standard scores for the adaptive domain scores and the General Adaptive Composite (GAC). The ABAS-2 includes 10 skill area scores (Communication, Community Use, Functional Academics, Health and Safety, Home or School Living, Leisure, Self-Care, Self-Direction, Social, and Work). The ABAS-2 has four domain composite scores (Conceptual, Social, Practical, and General Adaptive Composite or GAC). The GAC is derived from the sum of scaled scores from the skill areas and compares a person's global adaptive skills to the adaptive skills of others in the same age group from the standardization sample. The sum of scaled scores from the Communication, Functional Academics, and Self-Direction skill areas comprise the Conceptual domain. The sum of scaled scores from the Social and Leisure skill scores combine to make the Social domain. The sum of scaled scores from the Self-Care, Home or School Living, Community Use, Health and Safety, and Work comprise the Practical domain. The GAC and domain composite scores have a mean of 100 and a standard deviation of 15. Skill area standard scores have a mean of 10 and a standard deviation of 3 .

The ABAS-2 manual provides evidence of adequate reliability and validity (Harrison \& Oakland, 2003). Internal consistency coefficients of most of the skill areas are .90 or greater. Test-retest reliability yielded coefficients in the $.90 \mathrm{~s}$ for GAC scores, $.80 \mathrm{~s}$ to $.90 \mathrm{~s}$ for adaptive scores, and $.70 \mathrm{~s}$ to $.90 \mathrm{~s}$ for the individual skills areas. Inter-rater reliability was also measured and generally exceeded .80 whereas cross-informant coefficients generally exceeded .70 (Richardson \& Burns, 2005). Extensive information regarding the validity of the ABAS-2 is presented in the manual. The test demonstrates strong sensitivity to age group and for clinical populations. All forms had intercorrelations between skill areas between $.40 \mathrm{~s}$ to $.70 \mathrm{~s}$. In addition, the authors (Harrison \& Oakland, 2003) conducted several validation studies in order to demonstrate the concurrency between the ABAS-2 and other related scales. These scores ranged from a low of .70 to a high of .84 . Of particular interest to the current study is the correlation between the GAC on the ABAS-2 and the BASC Adaptive Skills Composite which was .80 (Harrison \& Oakland, 2003).

\subsubsection{The Social Skills Improvement Rating Scale (SSIS)}

The SSIS (Gresham \& Elliot, 2008) is a revision of the widely used SSRS (Social Skills Rating System) that provides information regarding the social skills, academic competence, and problem behavior of children and adolescents. It has a parent, teacher and student (preschool, younger students and older students) form. For the purposes of the current study, we utilized the parent and older student (ages 13 to 18) forms. On the parent form, raters responded to items about the frequency of behaviors using a 4 point Likert scale $(0=$ Never, $1=$ Seldom, $2=$ Often, and $3=$ Almost Always. The parent form is written at a $5^{\text {th }}$ grade reading level and the student forms are below a $2^{\text {nd }}$ grade reading level (Crosby, 2011). The SSIS provides standard scores for all composites with a mean of 100 and a standard deviation of 15. The Social Skills Composite includes Communication, Cooperation, Assertion, Responsibility, Empathy, Engagement, and Self-Control. The Problem Behaviors Composite includes the following sub-scales: Externalizing, Bullying, Hyperactivity/Inattention, Internalizing, and Autism Spectrum. 
Finally, the Academic Competence composite includes Reading Achievement, Math Achievement and Motivation to Learn. The SSIS also includes a Top 10 Scale that includes 10 social skills which teachers rated as being the most critical for academic success. These skills are as follows: 1) listens to others, 2) follows directions, 3) follows the rules, 4) ignores peer distractions, 5) asks for help, 6) takes turns in conversations, 7) cooperates with others, 8) controls temper in conflict situations, 9) acts responsibly when with others, and 10) shows kindness to others.

According to the SSIS manual (Gresham \& Elliot, 2008), median alpha levels for reliability estimates were at least mid to upper $.90 \mathrm{~s}$ for the Social Skills, Problem Behaviors and Academic Competence Scales. The subscales that comprise these composites had alpha estimates of at least .70. Median estimates were .80 for the parent form and closer to .80 for the student form. Test-retest reliability of the measure provides evidence of stability across administrations for both the parent (median coefficient $=.80$ ) and student (median coefficient $=.71$ ) forms. Results from validation studies demonstrated adequate validity with correlations of the scales measuring similar constructs being high and those measuring different constructs being low.

\subsubsection{The Behavior Assessment System for Children, Second Edition (BASC-2)}

The BASC-2 is a widely used norm-referenced diagnostic tool that uses a multidimensional approach to evaluate social, emotional, and behavioral functioning and self-perceptions of children aged 2 to 21 years (Reynolds \& Kamphaus, 2004). The BASC-2 is comprised of a Teacher Rating Scale (TRS), Parent Rating Scale (PRS), and a Self-Report of Personality (SRP) of behaviors and feelings. For the purpose of our study, we utilized the PRS and the SRP. The PRS is written at a $4^{\text {th }}$ grade reading level and contains questions measuring the child's adaptive skills and problem behaviors in the community/home setting. The format of the questions uses a four-choice Likert response scale of Never, Sometimes, Often, and Almost Always. Responses on the PRS yield scores on the following composite scales: Externalizing Problems, Internalizing Problems, Behavioral Symptom Index, and Adaptive Skills. The adolescent SRP (ages 12-21) was used for this study. It is written at a $3^{\text {rd }}$ grade reading level and contains some questions that assess the frequency of experiences (rated from $0=$ never to $3=$ almost always) and others are true/false (T/F) questions assessing the presence of thoughts and behaviors. The adolescent SRP yields the following composite scores: School Problems, Internalizing Problems, Inattention/Hyperactivity, Emotional Symptom Index and Personal Adjustment. Results are analyzed independently, reviewed in comparison with each other, and considered in light of established norms. Raw scores are converted to a T-score with a mean of 50 and a standard deviation of 10 . The BASC-2 is used by psychologists to measure and diagnose the presence of clinically related mental health and behavioral problems. High scores on the clinical scales indicate problems, whereas low scores on the adaptive scales indicate poor functioning.

The BASC-2 reliability was established based on the normed sample of a general and clinical population sample (Reynolds \& Kamphaus, 2004). Test-retest reliability was tested with individuals across all age groups who were part of both the general and clinical sample one to eight weeks following the first administration. Average correlations were in the .80 s for composite scores and between .70 and .80 for individual scores across age groups for the TRS, PRS and SRP. Test-retest reliabilities for the PRS were generally in the low .90s. The BASC-2 manual describes the test's validity and its' development. The PRS was compared to other behavioral measures and yielded correlations between subscales in the .70s and $.80 \mathrm{~s}$ when they addressed similar content. The adolescent and college scores on the SRP scale were correlated with a variety of self-report measures and yielded correlations in the $.50 \mathrm{~s}$ and $.60 \mathrm{~s}$ depending on the specific subscales that were compared.

\subsubsection{The Behavioral and Emotional Rating Scale, Second Edition (BERS-2)}

The BERS-2 is a widely used strength-based assessment instrument (Epstein, 2004) that helps to measure the personal strengths and competencies of children and adolescents including Interpersonal Strength, Involvement with Family, Intrapersonal Strength, School Functioning, Affective Strength, and Career Strength. The youth version contains 57 items and the parent version contains 52 items. Raters responded to each question on a 4-point Likert scale that ranged from 0 (not at all like me/the child) to 3 (very much like me/the child), which yields five subscales that have a mean standard score of 10 and a standard deviation of 3 . All scales are written at a fifth-grade reading level. The Interpersonal Strength subscale measures an adolescent's ability to control his or her emotions or behaviors in social situations (e.g., "I can express my anger in the right way"). The Family Involvement subscale measures a child's participation in and involvement with his or her family (e.g., "My family makes me feel wanted"). The School Functioning subscale measures competence in school and classroom tasks (e.g., "I complete tasks when asked"). The Intrapersonal Strength subscale measures an adolescent's outlook on his or her competence and accomplishments (e.g., "I believe in myself"). The Affective Strength 
subscale measures the ability of a child to accept affection from others and express feelings towards others (e.g., "It's okay when people hug me"). The BERS-2 also provides an overall Strength Quotient that provides a single summary score of strengths made up of all items with a reported internal consistency alpha of .95 and a test-retest reliability of .91. The sum of the subscale scores is converted into the Strength Index that has a mean of 100 and standard deviation of 15 .

The BERS-2 has established adequate reliability and validity (Epstein, 2004). Test-retest reliability was studied in the BERS-2: Parent Rating Scales (PRS) over a one-week period yielded coefficients that were at or above .80 (Mooney, Epstein, Ryser \& Pierce, 2005). A cross-informant reliability study comparing the agreement between the parent and youth forms, resulted in coefficients for the subscales and the overall strength quotient ranging from .50 to .63 (Synhorst, Buckley, Epstein, \& Ryser, 2005). A validation study on the BERS-2 found that the six BERS-2: PRS subscales and overall strength index were generally highly correlated with the composite scores from the parent forms of the Social Skills Rating System. Additionally, both the parent and youth versions of the BERS-2 were found to be negatively correlated with scales measuring deficits or problem scales (Buckley \& Epstein, 2004; Mooney et al., 2005).

\subsection{Procedure}

All participants were recruited at intake through a community - based youth mentoring program in Edmonton, Alberta. The participants had not begun any part of the intervention program when information was collected from them for the purpose of the current study. The adolescent participants completed the measures at the youth mentoring program. The time needed to complete all measures varied for each participant, however, were completed on average within 1-2 hours with breaks provided as needed. Their youth worker sat with them to aid them with any reading or comprehension difficulties. Each of the measures administered had a validity index. The validity indexes for all participants were within normal limits. Individuals trained in standard administration, applied the measures used in the current study. Test administrators read questions aloud and adolescents then proceeded by making their own response on forms provided. Items on measures were read aloud in order to ensure that adolescents understood the questions asked. Explanations related to word definitions and aid with comprehension was given by test administrators, however, no comments were made in terms of adolescent's endorsed responses. Due to the instability of family environment in the lives of adolescents with PAE, it is challenging to collect information from caregivers of this population. Therefore, the role of the caregiver raters varied for each participant and for each measure, and included the adolescent's biological mother or father, stepmother, adoptive mother, key/youth worker, guardian, grandmother / guardian, or person living with the adolescent. All of the participants who completed the parent-report forms were caregivers who had significant knowledge of the adolescent's day-to-day activities. Therefore, for some of the adolescents in the study, there were different caregivers who filled out the measures, however, they all completed parent-report forms for the ABAS-2, SSIS, BASC-2 and BERS-2. There were 17 caregivers who completed the ABAS-2, 20 who completed the SSIS, 18 who completed the BASC-2, and 23 who completed the BERS-2. There were 19 adolescents who completed the ABAS-2, 27 adolescents who completed the SSIS, 27 adolescents who completed the BASC-2, and 29 adolescents who completed the BERS-2. Only data collected at program entry (baseline) is used in this paper, thus the results reported do not measure the impact of the intervention.

\section{Results}

\subsection{Data Analyses}

Data was analyzed using PASW Statistics Version 18 for Windows. Descriptive statistics and one-sample independent t-tests were conducted to compare standard scores to the norm for adolescents and caregivers on all 4 measures (ABAS-2, SSIS, BASC-2, BERS-2). Paired sample t-tests were then performed between paired scores of corresponding scales for adolescent and caregiver on ABAS-2, SSIS, and BERS-2. The scales on the BASC-2 parent and adolescent version could not be compared using paired sample t-tests because they contain many different subscales. This study was exploratory in nature, and the goal was to identify potential interesting effects for future investigation. In addition, we did not have any specific hypothesis on which dependent variables should be different and which should not be different. Therefore, we interpreted each parent and caregiver score to each dependent variable (ABAS-2, SSIS, BASC-2, BERS-2) separately. For these reasons, we did not correct the alpha level for multiple t-tests. Furthermore, the correlations among the dependent variables (i.e., subscales) were low, so we were unable to proceed with multivariate analyses. Table 1 presents the means, standard deviations, and paired sample t-tests of composite scales on the four measures for caregivers and adolescents. 


\subsection{ABAS-2}

On the measure of adaptive skills we compared our sample to the norm mean of $100, \mathrm{SD}=15$ for all domain composites using one-sample independent t-tests. Scores that are lower than the mean indicate that adolescents have more difficulty in the area assessed. Skill area scaled scores were compared to the norm mean of $10, \mathrm{SD}=3$. Caregivers rated adolescents as significantly lower than the norm on all adaptive skills composites: GAC $(t(18)=$ -11.64, $\mathrm{p}<0.01)$, Conceptual $(t(18)=-12.16, \mathrm{p}<0.01)$, Social $(t(18)=-9.17, \mathrm{p}<0.01)$, and Practical $(t(17)=-7.10$, $\mathrm{p}<0.01)$. When compared to the norm, adolescent scores indicated that they rated themselves as significantly lower on the GAC composite $(t(18)=-3.54, \mathrm{p}<0.01)$, Conceptual composite $(t(18)=-4.54, \mathrm{p}<0.01)$, and the Practical composite $(t(18)=-2.61, \mathrm{p}<0.05)$. Adolescents did not rate themselves as significantly different from the mean on the Social composite ( $\mathrm{p}>0.05)$.

Paired sample t-tests were then conducted between caregivers and adolescents on the ABAS-2 which indicated that adolescents rated themselves as significantly higher than caregivers on scaled scores of Self-Care $(t(11)=$ 3.64, $\left.\mathrm{p}<0.01, \eta \rho^{2}=.546\right)$, Self-Direction $\left(t(11)=4.24, \mathrm{p}<0.01, \eta \rho^{2}=.620\right)$ and Social $(t(11)=5.29, \mathrm{p}<0.01)$. Adolescents also rated themselves as significantly higher on composites of GAC $\left(t(10)=2.89, \mathrm{p}<0.05, \eta \rho^{2}\right.$ $=.455)$, Conceptual $\left(t(11)=2.43, \mathrm{p}<0.05, \eta \rho^{2}=.350\right)$, and Social $\left(t(11)=3.23, \mathrm{p}<0.01, \eta \rho^{2}=.486\right)$. All other comparisons were non-significant, all ps $>0.12$. See Table 1 .

\subsection{SSIS}

On the measure of social skills we compared standard scores for composites of our sample to the norm mean of $100, \mathrm{SD}=15$ using one-sample independent t-tests. Lower scores on the Social Skills and Top-10 composite indicated that adolescents were having more difficulty in that area. Conversely, lower scores on the Problem Behavior composite indicated that adolescents were having less difficulty in that area. Caregivers rated adolescents as significantly worse than the norm on the following composites (see Table 1): Social Skills $(t(20)$ $=-11.54, \mathrm{p}<0.01)$, Problems Behaviors $(t(21)=10.86, \mathrm{p}<0.01)$ and Top 10 scale $(t(20)=-12.11, \mathrm{p}<0.01)$. Adolescents rated themselves as significantly worse than the norm on the Problem Behaviors composite $(t(26)=$ $4.85, \mathrm{p}<0.01)$. All other comparisons were non-significant, all $\mathrm{ps}>0.06$.

Paired sample t-tests were then conducted between caregivers and adolescents on the SSIS. On the Social Skills composite, caregivers rated adolescents as significantly lower than adolescents rated themselves, $t(16)=-6.09$, $\mathrm{p}<0.01, \eta \rho^{2}=.698$. On the Problem Behaviors composite, caregivers rated adolescents as having significantly more problem behaviors than adolescents rated themselves, $t(18)=6.45, \mathrm{p}<0.01, \eta \rho^{2}=.698$. On the Top-10 scale, caregivers rated adolescents as significantly lower than adolescents rated themselves, $t(16)=-4.52, \mathrm{p}<0.01$, $\eta \rho^{2}=.561$, indicating that caregivers viewed adolescents as possessing less critical skills for classroom success than adolescents do.

\subsection{BASC-2}

On BASC-2 we compared t-scores from subscales and composite scales of both the self-report and parent form of our sample population to the norm mean of $50, \mathrm{SD}=10$ using one-sample independent t-tests. High scores on the clinical scales indicated problem areas, whereas low scores on the adaptive scales indicated poor functioning. Caregivers' scores were all significantly higher than the mean on all clinical scales and lower than the mean on all adaptive scales, but all other comparisons were non-significant, $\mathrm{ps}>0.03$. On the composite scales, caregivers rated adolescents as significantly worse than the norm on Externalizing Problems $(t(21)=7.21, \mathrm{p}<0.01)$, Internalizing Problems $(t(21)=3.52, \mathrm{p}<0.01)$, Behavior Symptom Index $(t(21)=7.61, \mathrm{p}<0.01)$, and Adaptive Skills $(t(21)=-7.67, \mathrm{p}<0.01)$. Adolescents rated themselves as significantly worse than the norm on the clinical composites of School Problems $(t(26)=3.21, \mathrm{p}<0.01)$ and Inattention/Hyperactivity $(t(26)=2.26, \mathrm{p}<0.05)$. All other comparisons were non-significant, all ps>0.07. See Table 1. There were no paired sample t-tests conducted on the BASC-2 because the self - report of adolescent and parent scales consisted of many different scales and composites, which are not comparable to each other.

\subsection{BERS-2}

On the measure assessing behavioral and emotional strengths of adolescents, we compared scaled subscale scores of our sample to the norm mean of $10, \mathrm{SD}=3$ using one-sample independent t-tests. We also compared the Strength Index using one-sample independent t-tests with the norm mean of 100 and standard deviation of 15. Higher scores indicated more strength in the area being assessed. Caregivers rated adolescents as significantly worse than the mean on Interpersonal Strength $(t(22)=-9.88, \mathrm{p}<0.01)$, Family Involvement $(t(22)=-12.23$, $\mathrm{p}<0.01)$, Intrapersonal Strength $(t(22)=-12.016, \mathrm{p}<0.01)$, School Functioning $(t(22)=-12.44, \mathrm{p}<0.01)$ and Affective Strength $(t(22)=-8.18, \mathrm{p}<0.01)$. In addition Career Strength of adolescents was rated by caregivers as 
significantly lower than the mean $(t(19)=-2.77, \mathrm{p}=0.012)$. Caregivers also rated adolescents as significantly lower from the mean on the Strength Index $(t(22)=-8.65, \mathrm{p}<0.01)$ of the BERS-2. Adolescents rated themselves as significantly worse than the mean on Family Involvement $(t(28)=-2.93, \mathrm{p}<0.01)$ and School Functioning $(t(28)=-2.26, \mathrm{p}<0.05)$. Conversely, they rated themselves as significantly better than the mean on the Affective Strength scale $(t(28)=2.12, \mathrm{p}<0.05)$. The adolescent mean score on the Strength Index was not significantly different from the norm mean $(t(28)=-1.535, \mathrm{p}=0.136)$ although they did rate themselves as having a lower Strength Index when compared to the mean. All other comparisons were non-significant, all ps $>0.66$. Paired sample t-tests were conducted between caregivers and adolescents on measures of the BERS-2 which indicated that adolescents rated themselves significantly higher than caregivers on Interpersonal Strength $(t(20)=-4.93$, $\left.\mathrm{p}<0.01, \eta \rho^{2}=.549\right)$, Family Involvement $\left(t(20)=-5.67, \mathrm{p}<0.01, \eta \rho^{2}=.617\right)$, Intrapersonal Strength $(t(20)=$ $\left.-5.66, \mathrm{p}<0.01, \eta \rho^{2}=.615\right)$, School Functioning $\left(t(20)=-4.28, \mathrm{p}<0.01, \eta \rho^{2}=.478\right)$ and Affective Strength $(t(20)$ $\left.=-8.40, \mathrm{p}<0.01, \eta \rho^{2}=.779\right)$. The comparison between caregivers and adolescents on the measure of Career Strength was non-significant, $\mathrm{p}>0.101$ ).

Table 1. Means, standard deviations and paired sample t-tests on the ABAS-2, SSIS, BASC-2 and BERS-2 for caregiver and adolescent

\begin{tabular}{|c|c|c|c|c|c|}
\hline \multicolumn{6}{|c|}{ Rater } \\
\hline \multirow[t]{2}{*}{ Measure } & \multicolumn{2}{|c|}{ Caregiver (C) } & \multicolumn{2}{|r|}{ Youth (Y) } & \multirow{2}{*}{$\begin{array}{c}\text { C-Y Comparison } \\
\mathrm{p}\end{array}$} \\
\hline & $\mathrm{N}$ & $\mathrm{M}(\mathrm{SD})$ & $\mathrm{N}$ & $\mathrm{M}(\mathrm{SD})$ & \\
\hline \multicolumn{6}{|l|}{ ABAS-2 } \\
\hline GAC & 19 & $67.68(12.09)$ & 19 & $87.47(15.41)$ & $<.05$ \\
\hline Conceptual & 19 & $71.47(10.22)$ & 19 & $85.68(13.74)$ & $<.05$ \\
\hline Social & 19 & $73.53(12.58)$ & 19 & $93.00(15.47)$ & $<.01$ \\
\hline Practical & 18 & $70.11(17.85)$ & 19 & $89.26(17.94)$ & $\mathrm{ns}$ \\
\hline \multicolumn{6}{|l|}{ SSIS } \\
\hline Social Skills & 21 & $69.76(12.01)$ & 26 & $94.54(16.21)$ & $<.01$ \\
\hline Problem Behaviors & 22 & $131.59(13.64)$ & 27 & $144.04(15.03)$ & $<.01$ \\
\hline Top 10 scale & 21 & 69.86(11.40) & 25 & $93.08(17.68)$ & $<.01$ \\
\hline \multicolumn{6}{|l|}{ BASC-2 } \\
\hline Externalizing Problems & 22 & $67.95(11.68)$ & & & $\mathrm{n} / \mathrm{a}$ \\
\hline Internalizing Problems & 22 & $59.73(12.95)$ & 27 & $54.74(13.17)$ & $\mathrm{n} / \mathrm{a}$ \\
\hline Behavior Symptom Index & 22 & $65.64(9.64)$ & & & $\mathrm{n} / \mathrm{a}$ \\
\hline School Problems & & & 27 & $56.93(11.20)$ & $\mathrm{n} / \mathrm{a}$ \\
\hline Adaptive Skills & 22 & $38.14(7.25)$ & & & $\mathrm{n} / \mathrm{a}$ \\
\hline Inattention/Hyperactivity & & & 27 & $56.15(14.13)$ & $\mathrm{n} / \mathrm{a}$ \\
\hline Emotional Symptoms Index & & & 27 & $52.67(13.16)$ & $\mathrm{n} / \mathrm{a}$ \\
\hline Personal Adjustment & & & 27 & 48.11(11.67) & $\mathrm{n} / \mathrm{a}$ \\
\hline \multicolumn{6}{|l|}{ BERS-2 } \\
\hline Interpersonal Strength & 23 & $6.39(1.75)$ & 29 & $10.28(3.48)$ & $<.01$ \\
\hline Family Involvement & 23 & $6.09(1.53)$ & 29 & $8.34(3.03)$ & $<.01$ \\
\hline Intrapersonal Strength & 23 & $6.17(1.52)$ & 29 & $9.90(3.37)$ & $<.01$ \\
\hline School Functioning & 23 & $5.39(1.77)$ & 29 & $8.59(3.36)$ & $<.01$ \\
\hline Affective Strength & 23 & $6.74(1.91)$ & 29 & $11.03(2.62)$ & $<.01$ \\
\hline Career Strength & 23 & $8.60(2.25)$ & 29 & $9.73(3.08)$ & ns \\
\hline Strength Index & 23 & $70.57(16.31)$ & 29 & $94.10(20.68)$ & $\mathrm{n} / \mathrm{a}$ \\
\hline
\end{tabular}


Note. On the ABAS-2, lower scores are worse; on the SSIS, lower scores on Social Skills and Top-10 composites are worse and higher scores on Problem Behaviors are worse; on the BASC-2, for all composites higher scores are worse, however, for the Adaptive Skills and Personal Adjustment composites, lower score are worse; on the BERS-2, higher scores are better.

$\mathrm{p}=$ 2-tailed paired sample t-tests

\section{Discussion}

The goal of this study was to examine the characteristics and experiences of adolescents prenatally exposed to alcohol, as viewed by the adolescents and their caregivers. Measures of adaptive behavior (ABAS-2), social skills (SSIS), mental health (BASC-2), and personal strength (BERS-2) were completed by caregivers and adolescents to provide information on the functioning of each adolescent.

On the measure of adaptive behavior (measures with the ABAS-2) adolescents and caregivers perceived adolescents' daily living skills as a problem area across most of the domains assessed. Caregivers viewed adolescents' adaptive skills as an area of significant concern, which is congruent with other research (Kaemingk \& Paquette, 1999; Streissguth et al., 2004) that reports adaptive functioning deficits among individuals with PAE as well as increasing difficulty with social relationships as they age (Steinhausen \& Spohr, 1998; Streissguth et al., 1991). Adolescents however, described fewer concerns, especially in the area of social functioning, possibly reflecting a desire to present themselves in a more positive light, or an unwillingness to admit to difficulties (Karver, 2006). However, it could also mean that although adolescents with PAE recognize there is a problem, they do not appreciate the degree to which it exists as perceived by others. Although this may not be entirely surprising in the adolescent period, the extent to which this lack of insight exists may reflect the compounded effect of typical adolescent egocentrism and the impact of alcohol on the developing brain. For instance, the frontal cortex (involved in executive functioning) is negatively affected by PAE (see Rasmussen, 2005 for a review) and is associated with the development of self-awareness (Majovski \& Breiger, 2009). Adolescents' lack of awareness or perspective of their social, emotional, behavioral, and adaptive functioning could be affected by deficits in executive functioning and metacognition. Problems with judgment, comprehension, and abstraction (Streissguth et al., 1991) can also affect the self-perception of an adolescent with PAE. This can explain the lack of insight that adolescents have into their own challenges and difficulties.

In terms of mental health (measured with the BASC-2), caregivers again reported concerns in most areas with the exception of anxiety and somatization. This is consistent with the high rates of co-morbid psychiatric diagnoses among individuals with PAE (Pei et al., 2011), although surprising to see the low rate of reported anxiety concerns given the high rate of anxiety disorders reported for this population (Olson et al. 2009). It is also noteworthy that it was the externalizing concerns, such as antisocial behavior and aggression that were described as the greatest concerns, as opposed to internalizing disorders. It could be these behaviors are simply more easily detected by the caregivers, and therefore overshadow the more subtle presentation of internalizing disorders such as anxiety and somatization. Adolescents also did not perceive anxiety and somatization as areas of concern. However they also did not report the same amount of externalizing concerns as their caregivers. Most interesting in the adolescents' reports was that they perceive their functioning to be outside of their own control. The perception that these adolescents hold of not having the capacity to change events in their own lives may reflect the degree of social support they have had throughout their lives, or their own understanding of how their brain injury impacts them. Regardless, it is clear they feel out of control of their own behavior, which could have a significant impact on the way in which they engage with systems of support and the responsibility they take for their own behavior-good and bad. Researchers in the area of predicting health related behaviors have found a link between high levels of self-efficacy and motivation to change health-related behaviors (Schwarzer \& Renner, 2000) suggesting that intervention efforts with these youth may need to be increasingly intentional in efforts to build self-efficacy by increasing opportunities for autonomy and decision making in safe contexts.

In considering their social skills (measured with the SSIS), adolescents reported significantly more problems than the norm sample. In particular they reported concerns with their own acting out behavior (externalizing), bullying, Hyperactivity/Inattention, Internalizing, and unusual or idiosyncratic behavior. This may reflect the fact that behaviors captured on these indexes tend to be more easily identified as they are more concrete and require less introspection. Their recognition of deficits in these areas may also reflect the feedback they receive by way of external consequences. Additionally, it is possible that adolescents view these problems in a more favourable light in order to maintain a positive self-image of being outwardly strong and tough (Copes \& Williams, 2007) and therefore, more readily report these concerns. The adolescents in this study also rated 
themselves as significantly worse than the norm on School Problems and Inattention/Hyperactivity on the BASC-2. These results are consistent with other research, which has shown that academic difficulties as well as ADHD (Olson et al., 2009) are a significant area of concerns among adolescents with PAE, and may again reflect greater awareness of behavior that is external, concrete, and often the subject of external feedback (e.g. consequences).

On the measures of personal strengths (measured with the BERS-2), caregivers rated adolescents as significantly worse than their peers in most areas of daily functioning. Specifically, caregivers viewed adolescents as having significantly more difficulty with their ability to control their emotions and behavior in social situations, participation and relations to their family, competence and accomplishments, competence in classroom tasks, and their ability to give and receive affect. These difficulties may be related to deficits in inhibitory control commonly found among adolescents with PAE (Fryer et al., 2007). One possible explanation for the high level of reported problems by caregivers in the present study could be due to the fact that living and caring for an adolescent with PAE is a demanding and stressful job (Giunta \& Streissguth, 1988; Paley et al., 2005; 2006) and therefore parental stress may have exacerbated some of the scores on these measures. In contrast, adolescents did not identify these concerns and perceived themselves as functioning much more effectively in these social arenas. This difference could be due to a host of different reasons including effects of PAE on the brain as well as the adolescents' perception of having little control over the events that occur in their lives.

Both caregivers and adolescents were able to identify strength in their perception for the future of the youth. Despite all the identified areas of concern that were reported by both groups, all remain optimistic about the adolescents' future. This is an important finding as it identifies a strength that both caregivers and adolescents share concerning the adolescents' career and vocational goals. It is a welcome surprise that despite all the cognitive, emotional and behavioral challenges that individuals face as a result of prenatal exposure to alcohol; there is a sense of hope from both caregivers and adolescents about the future. A parent's sense of optimism towards their child with PAE can serve as a protective factor (Olson et al., 2009). Therefore, caregivers' optimistic view of the future can be helpful in aiding and supporting the adolescent to achieve future goals.

\subsection{Implications for Research, Policy and Practice}

It is evident that caregivers and adolescents with PAE and/or FASD view the adolescent's functioning differently. Therefore, when conducting research as well as working with this population, it will be important to use measures that allow data to be collected from multiple perspectives, as well as recognize the different perspectives. Recognizing that an adolescents' apparent indifference may actually reflect lack of insight could change the approaches used. It may also be beneficial to begin by addressing areas of concern that the adolescents' identity, and possibly acknowledging their experience of feeling a lack of control over the direction their lives, and the effects of their behavior on others. Reclassifying our understanding of the behavior problems exhibited by these adolescents as due to, at least in part, prenatal brain injury, rather than defiance or antisocial desires alone, may help to build supports and advocacy, as well as understanding and compassion. Our findings identified areas of agreement between caregiver and adolescent. This finding was found on the measure that identified strengths in the adolescents. The assessment of strengths as opposed to deficits can facilitate more positive discussions and help to improve upon the strengths, competencies and overall well-being of the adolescent. The knowledge regarding the strengths identified by the adolescent and those around them can be a useful starting point to begin discussions when planning an intervention with an adolescent with PAE that is future and career oriented.

\subsection{Limitations of Study}

This study makes a significant contribution to how we measure perceptions and highlights the importance of using multiple measures with multiple perspectives in PAE and FASD research. There were a number of limitations in our study; one is the small sample size of program participants and caregivers. Although there have been studies where larger sample sizes have been used (Alati et al., 2006; Barr et al., 2006; O'Connor, McCracken, \& Best, 2006), small sample sizes continue to be a limitation within the FASD field. Recruiting and retaining participants in research studies who have PAE is challenging due to the inconsistency in caregivers and the instability of the adolescents' home environment. Therefore, researchers report in the literature studies that have been conducted with smaller sample sizes than other populations. Another limitation is that all scales and ratings are subject to bias.

We did not assess the intellectual ability of the adolescents, which could be seen as a limitation due to the widespread cognitive deficits present among this population (Mattson \& Riley, 1998), however research has shown that IQ is not the best predictor of functional outcomes in FASD (Thomas et al., 1998). Future research 
examining how cognitive functioning in adolescents with PAE and/or FASD influence their perceptions is warranted. In addition, some of the discrepancies between results from adolescents versus caregivers could be due to speech and language difficulties commonly seen in PAE (Stade et al., 2006). The adolescents' responses to questions may reflect a difference in perception or comprehension.

The lack of control group in our study is another limitation, however because the sample was from a community-based mentoring program for youth with PAE and FASD who were at risk, there are many variables that are difficult to control for in this population such as SES, IQ, negative life experiences etc. Thus we were unable to test a similar at-risk group to be included as a non-exposed control group. Furthermore, because the sample was already at-risk and referred for the mentoring program they may not be representative of the entire PAE or FASD population and may have more areas of need. Nevertheless, the current study provides a better understanding of the way adolescents with PAE and their caregivers perceive the adolescents' worlds. These findings will help inform discussion and dialogue when working with this population. We anticipate that this ongoing research will reveal valuable information on the adaptive, mental health and social skill functioning of adolescents with PAE.

\section{Conclusions}

The study highlights the differences in perceptions between caregivers and adolescents: All caregivers perceived adolescents as having greater difficulty with adaptive, emotional, and social functioning compared to the perception of the adolescents themselves. Although adolescents also described many areas of difficulty, they often did so to a much lesser extent than their caregivers. Caregivers and adolescents agreed that they see potential for the adolescents' future despite current difficulties. Further research in this area can help clarify these results in order to determine the concerns of those living and working with an adolescent with PAE and as well as the adolescents themselves.

\section{Acknowledgements}

We would like to express a special thanks to the youth and caregivers who participated in this study.

\section{References}

Alati, R., Mamun, A. A., Williams, G. M., O’Callaghan, M., Najman, J. M., \& Bor, W. (2006). In utero alcohol exposure and prediction of alcohol disorders in early adulthood: A birth cohort study. Archives of General Psychiatry, 63, 1009-1015. http://dx.doi.org/10.1001/archpsyc.63.9.1009

Ase, F., Ilone, A. R., Mirijam, K., Pekka, S., Eugene, H. H., Sarah, M. N., \& Marit, K. (2012). Adaptive behaviour in children and adolescents with foetal alcohol spectrum disorders: a comparison with specific learning disability and typical development. European Child \& Adolescent Psychiatry, 21(4), 221-231. http://dx.doi.org/10.1007/s00787-012-0256-y

Astley, S. J. (2004). Diagnostic guide for fetal alcohol spectrum disorders: The 4-digit diagnostic code (3rd ed.). Seattle, WA: University of Washington Publication Services.

Barr, H. M., Bookstein, F. L., O’Malley, K. D., Connor, P. D., Huggins, J. E., \& Streissguth, A. P. (2006). Binge drinking during pregnancy as a predictor of psychiatric disorders on the structured clinical interview for DSM-IV in young adult offspring. American Journal of Psychiatry, 163(6), 1061-1065. http://dx.doi.org/10.1176/appi.ajp.163.6.1061

Buckley, J. A., \& Epstein, M. H. (2004). The behavioral and emotional rating scale-2 (BERS-2): Providing a comprehensive approach to strength-based assessment. The California School Psychologist, 9, 21-27.

Chudley, A. E., Conry, J., Cook, J. L., Loock, C., Rosales, T., \& LeBlanc, N. (2005). Fetal alcohol spectrum disorder: Canadian guidelines for diagnosis. Canadian Medical Association Journal, 172(5-Suppl.), S1-S21. http://dx.doi.org/10.1503/cmaj.1040302

Copes, H., \& Williams, J. P. (2007). Techniques of affirmation: Deviant behavior, moral, commitment, and subcultural identity. Deviant Behavior, 28(3), 247-272. http://dx.doi.org/10.1080/01639620701233167

Crocker, N., Vaurio, L., Riley, E. P., \& Mattson, S. N. (2009). Comparison of adaptive behavior in children with heavy prenatal alcohol exposure or attention-deficit/hyperactivity disorder. Alcoholism: Clinical and Experimental Research, 33(11), 2015-2023. http://dx.doi.org/10.1111/j.1530-0277.2009.01040.x

Crosby, J. W. (2011). Test review: F. M. Gresham, \& S. N. Elliott social skills improvement rating scales. Journal of Psychoeducational Asssessment, 29(3), 292-296. http://dx.doi.org/10.1177/0734282910385806

Epstein, M. H. (2004). Behavioral and Emotional Rating Scale-2: A strength-based approach to assessment. 
Austin, TX: PRO-ED.

Fryer, S. L., Tapert, S. F., Mattson, S. N., Paulus, M. P., Spadoni, A. D., \& Riley, E. P. (2007). Prenatal alcohol exposure affects frontal-striatal BOLD response during inhibitory control. Alcoholism: Clinical and Experimental Research, 31(8), 1415-1424. http://dx.doi.org/10.1111/j.1530-0277.2007.00443.x

Gresham, F. M., \& Elliott, S. N. (2008). Rating Scales Manual. SSIS Social Skills Improvement System. Minneapolis, Pearson.

Giunta, C. T., \& Streissguth, A. P. (1988). Patients with fetal alcohol syndrome and their caretakers. Social Casework, 69(7), 453-459.

Green, J. H. (2007). Fetal alcohol spectrum disorders: Understanding the effects of prenatal alcohol exposure and supporting students. Journal of School Health, $103-108$. http://dx.doi.org/10.1111/j.1746-1561.2007.00178.x

Harrison, P. L., \& Oakland T. (2003). Adaptive Behavior Assessment System (2nd ed.). San Antonio, TX: Harcourt Assessment.

Kaemingk, K., \& Paquette, A. (1999). Effects of prenatal alcohol exposure on neuropsychological functioning. Developmental Neuropsychology, 15(1), 111-140. http://dx.doi.org/10.1080/87565649909540741

Karver, M. S. (2006). Determinants of multiple informant agreement on child and adolescent behavior. Journal of Abnormal Child Psychology, 34(2), 251-262. http://dx.doi.org/10.1007/s10802-005-9015-6

Kodituwakku, P. W. (2007). Defining the behavioral phenotype in children with fetal alcohol spectrum disorders: A review. Neuroscience \& Biobehavioral Reviews, 31(2), $192-201$. http://dx.doi.org/10.1016/j.neubiorev.2006.06.020

Kodituwakku, P. W. (2009). Neurocognitive profile in children with fetal alcohol spectrum disorders. Developmental Disabilities Research Reviews, 15(3), 218-224. http://dx.doi.org/10.1002/ddrr.73

Kully-Martens, K., Denys, K., Treits, S., Tamana, S., \& Rasmussen, C. (2012). A review of social skills deficits in individuals with fetal alcohol spectrum disorders and prenatal alcohol exposure: Profiles, mechanisms, and interventions. Alcoholism: Clinical and Experimental Research, 36, 568-576. http://dx.doi.org/10.1111/j.1530-0277.2011.01661.x

Majovski, L., \& Breiger, D (2009). Development of higher brain functions: Birth through adolescence. In C. R. Reynolds, \& E. Fletcher-Janzen (Eds.), Handbook of Clinical Child Neuropsychology (pp. 67-116). New York, NY: Springer. http://dx.doi.org/10.1007/978-0-387-78867-8_4

Mattson, S. N., Crocker, N., \& Nguyen, T. T. (2011). Fetal alcohol spectrum disorders: Neuropsychological and behavioral features. Neuropsychology Review, 21(2), 81-101. http://dx.doi.org/10.1007/s11065-011-9167-9

Mattson, S. N., \& Riley, E. P. (1998). A review of the neurobehavioral deficits in children with fetal alcohol syndrome or prenatal exposure to alcohol. Alcoholism: Clinical and Experimental Research, 22(2), $279-294$. http://dx.doi.org/10.1111/j.1530-0277.1998.tb03651.x

Mooney, P., Epstein, M. H., Ryser, G., \& Pierce, C. D. (2005). Reliability and Validity of the Behavioral and Emotional Rating Scale-Second Edition: Parent Rating Scale. Children \& Schools, 27(3), 147-155. http://dx.doi.org/10.1093/cs/27.3.147

O'Connor, M. (2001). Prenatal alcohol exposure and infant negative affect as precursors of depressive features in children. Infant Mental Health Journal, 22(3), 291-299. http://dx.doi.org/10.1002/imhj.1002

O’Connor, M. J., McCracken, J. T., \& Best, A. (2006). Under recognition of prenatal alcohol exposure in a child inpatient psychiatric setting. Mental Health Aspects of Developmental Disabilities, 9, 105-109.

O’Connor, M. J., Shah, B., Whaley, S., Cronin, P., Gunderson, B., \& Graham, J. (2002). Psychiatric illness in a clinical sample of children with prenatal alcohol exposure. The American Journal of Drug and Alcohol Abuse, 28(4), 743-754. http://dx.doi.org/10.1081/ADA-120015880

Olson, H. C., Oti, R., Gelo, J., \& Beck, S. (2009). Family matters: Fetal alcohol spectrum disorders and the family. Developmental Disabilities Research Reviews, 15(3), 235-249. http://dx.doi.org/10.1002/ddrr.65

Paley, B., O’Connor, M. J., Frankel, F., \& Marquardt, R. (2006). Predictors of stress in parents of children with fetal alcohol spectrum disorders. Journal of Developmental \& Behavioral Pediatrics, 27(5), $396-404$. http://dx.doi.org/10.1097/00004703-200610000-00005

Paley, B., O’Connor, M. J., Kogan, N., \& Findlay, R. (2005). Prenatal alcohol exposure, child externalizing 
behavior, and maternal stress. Parenting: Science and Practice, 5(1), 29-56. http://dx.doi.org/10.1207/s15327922par0501_2

Pei, J., Denys, K., Hughes, J., \& Rasmussen, C. (2011). Mental health issues in fetal alcohol spectrum disorder. Journal of Mental Health, 1-11. http://dx.doi.org/10.3109/09638237.2011.577113

Rasmussen, C. (2005). Executive functioning and working memory in fetal alcohol spectrum disorder. Alcoholism: Clinical and Experimental Research, 29(8), 1359-1367. http://dx.doi.org/10.1097/01.alc.0000175040.91007.d0

Rasmussen, C., Andrew, G., Zwaigenbaum, L., \& Tough, S. (2008). Neurobehavioral outcomes of children with fetal alcohol Spectrum disorder: A Canadian perspective. Paediatrics \& Child Health, 13(3), 185-191. Retrieved from http://www.pulsus.com/journals/journalHome.jsp?sCurrPg=journal\&jnlKy=5\&fold=Home

Reynolds, C. R., \& Kamphaus, R. W. (2004). Behavior Assessment System for Children (2nd ed.). Circle Pines, MN: American Guidance Service Publishing.7

Richardson, R. D., \& Burns, M. K. (2005). Adaptive behavior assessment system. Assessment for Effective Intervention, 30(4), 51-54. http://dx.doi.org/10.1177/073724770503000407

Schwarzer, R., \& Renner, B. (2000). Social-cognitive predictors of health behavior: Action self-efficacy and coping self-efficacy. Health Psychology, 19(5), 487-495. http://dx.doi.org/10.1037/0278-6133.19.5.487

Spohr, H.-L., Willms, J., \& Steinhausen, H. (2007). Fetal Alcohol Spectrum Disorders in young adulthood. The Journal of Pediatrics, 150(2), 175-179.e1. http://dx.doi.org/10.1016/j.jpeds.2006.11.044

Spohr, H.-L., \& Steinhausen, H. (1987). Follow-up studies of children with fetal alcohol syndrome. Neuropediatrics, 18, 13-17. http://dx.doi.org/10.1055/s-2008-1052428

Stade, B. C., Stevens, B., Ungar, W.J., Beyene, J., \& Koren, G. (2006). Health-related quality of life of Canadian children and youth prenatally exposed to alcohol. Health and Quality of Life Outcomes, 4(81), 1-10. http://dx.doi.org/10.1186/1477-7525-4-81

Stade, B., Ali, A., Bennett, D., Campbell, D., Johnston, M., \& Koren, G. (2009). The burden of prenatal exposure to alcohol: Revised measurement of cost. Canadian Journal of Clinical Pharmacology, 16(1), e91-e102. Retrieved from http://www.cjcp.ca.login.ezproxy.library.ualberta.ca

Steinhausen, H.-C., \& Spohr, H.-L. (1998). Long-term outcome of children with fetal alcohol syndrome: Psychopathology, behavior, and intelligence. Alcoholism: Clinical and Experimental Research, 22(2), 334-338. http://dx.doi.org/10.1111/j.1530-0277.1998.tb03657.x

Streissguth, A. P. (1997). Fetal Alcohol Syndrome: A guide for families and communities. Baltimore, MD: Paul $\mathrm{H}$ Brookes Publishing.

Streissguth, A. P., Sampson, P. D., Olson, H. C., Bookstein, F. L., Barr, H. M., Scott, M., ... Mirsky, A. F. (1994). Maternal drinking during pregnancy: Attention and short-term memory in 14-year-old offspring-a longitudinal prospective study. Alcoholism: Clinical and Experimental Research, 18(1), 202-218. http://dx.doi.org/10.1111/j.1530-0277.1994.tb00904.x

Streissguth, A. P., \& O’Malley, K. (2000). Neuropsychiatric implications and long-term consequences of fetal alcohol spectrum disorders. Seminars in Clinical Neuropsychiatry, 5(3), 177-190. http://dx.doi.org/10.1053/scnp.2000.6729

Streissguth, A. P., Aase, J., Clarren, S., Randels, S. P., LaDue, R., \& Smith, D. F. (1991). Fetal alcohol syndrome in adolescents and adults. The Journal of the American Medical Association, 265(15), 1961-1967. http://dx.doi.org/10.1001/jama.1991.03460150065025

Streissguth, A. P., Bookstein, F. L., Barr, H. M., Sampson, P. D., O’Malley, K., \& Young, J. K. (2004). Risk factors for adverse life outcomes in fetal alcohol syndrome and fetal alcohol effects. Journal of Developmental \& Behavioral Pediatrics, 25(4), 228-238. http://dx.doi.org/10.1097/00004703200408000-00002

Synhorst, L. L., Buckley, J. A., Reid, R., Epstein, M. H., \& Ryser, G. (2005). Cross informant agreement of the behavioral and emotional rating scale-2nd edition (BERS-2) Parent and youth rating scales. Child \& Family Behavior Therapy, 27(3), 1-11. http://dx.doi.org/10.1300/J019v27n03_01

Thomas, S. E., Kelly, S. J., Mattson, S. N., \& Riley, E. P. (1998). Comparison of social abilities of children with fetal alcohol syndrome to those of children with similar IQ scores and normal controls. Alcoholism: Clinical 
and Experimental Research, 22(2), 528-533. http://dx.doi.org/10.1111/j.1530-0277.1998.tb03684.x

Whaley, S., O'Connor, M., \& Gunderson, B. (2001). Comparison of the adaptive functioning of children prenatally exposed to alcohol to a nonexposed clinical sample. Alcoholism: Clinical and Experimental Research, 25(7), 1018-1024. http://dx.doi.org/10.1111/j.1530-0277.2001.tb02311.x

\section{Copyrights}

Copyright for this article is retained by the author(s), with first publication rights granted to the journal.

This is an open-access article distributed under the terms and conditions of the Creative Commons Attribution license (http://creativecommons.org/licenses/by/3.0/). 